\title{
Characterization of exosome-like
} vesicles derived from Taenia pisiformis

\section{cysticercus and their immunoregulatory role on macrophages}

\author{
Li-Qun Wang ${ }^{1}$, Ting-Li Liu' ${ }^{1}$, Pan-Hong Liang ${ }^{1}$, Shao-Hua Zhang ${ }^{1}$, Tao-Shan Li', Yan-Ping Li ${ }^{1}$, Guang-Xue Liu', \\ Li MaO ${ }^{1}$ and Xue-Nong Luo ${ }^{1,2^{*}}$
}

\begin{abstract}
Background: Taenia pisiformis is one of the most common intestinal parasites in canines, and leads to serious economic losses in the rabbit breeding industry. Exosome-like vesicles from parasites play crucial roles in host-parasite interactions by transferring cargo from parasites to host cells and by modulating host immunological response through inducing production of host-derived cytokines. Nevertheless, the mechanism by which exosome-like vesicles from T. pisiformis cysticercus regulate the macrophage immune response remains unknown.

Methods: Using ultracentrifugation, we isolated exosome-like vesicles from excretory/secretory products (ESP) of T. pisiformis cysticercus. The morphology and size of purified vesicles were confirmed by transmission electron microscopy (TEM) and nanoparticle tracking analysis (NTA). The components of proteins and miRNAs within these vesicles were identified by proteomic analysis and high-throughput small RNA sequencing. The biological function of targets of exosomal miRNAs was predicted by Kyoto Encyclopedia of Genes and Genomes (KEGG) pathway analysis. Moreover, the expression of Th1- and Th2-type immune response associated cytokines in RAW264.7 macrophages were evaluated by qPCR and ELISA. We found that exosome-like vesicles were typical cup-shaped vesicles with diameters from 30 to $150 \mathrm{~nm}$. A total of 87 proteins were identified by proteomic analysis, including proteins prominently associated with exosome-like vesicles biogenesis and vesicle trafficking. 41 known miRNAs and 18 novel miRNAs were identified in the exosome-like vesicles. Eleven selected miRNAs, including 7 known miRNAs (miR-71-5p, miR-10a-5p, miR-let7-5p, miR-745-3p, miR-219-5p, miR-124-3p and miR-4989-3p) and 4 novel miRNAs (novel-mir-3, novel-mir-7, novelmir-8 and novel-mir-11) were validated to exist in metacestiodes and exosome-like vesicles of T. pisiformis cysticercus by qPCR. The functions of most targets of exosomal miRNAs were mainly associated with signal transduction and the immune system. Additionally, T. pisiformis cysticercus-derived vesicles induced the production of IL-4, IL-6, IL-10, IL-13 and Arg-1, but downregulated the expression of IL-12, IFN- $\gamma$ and iNOS in RAW264.7 macrophages.
\end{abstract}

Conclusions: We demonstrated that proteins and miRNAs enclosed within exosome-like vesicles from T. pisiformis cysticercus have immunomodulatory functions. Furthermore, exosome-like vesicles were shown to induce the

\footnotetext{
*Correspondence: luoxuenong@caas.cn

1 State Key Laboratory of Veterinary Etiological Biology, Key Laboratory of Veterinary Parasitology of Gansu Province, Lanzhou Veterinary Research Institute, Chinese Academy of Agricultural Sciences, Lanzhou 730046, Gansu Province, People's Republic of China

Full list of author information is available at the end of the article
}

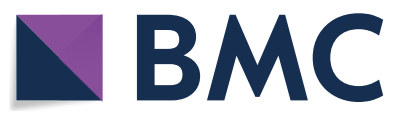

(c) The Author(s) 2020. This article is licensed under a Creative Commons Attribution 4.0 International License, which permits use, sharing, adaptation, distribution and reproduction in any medium or format, as long as you give appropriate credit to the original author(s) and the source, provide a link to the Creative Commons licence, and indicate if changes were made. The images or other third party material in this article are included in the article's Creative Commons licence, unless indicated otherwise in a credit line to the material. If material is not included in the article's Creative Commons licence and your intended use is not permitted by statutory regulation or exceeds the permitted use, you will need to obtain permission directly from the copyright holder. To view a copy of this licence, visit http://creativecommons.org/licenses/by/4.0/. The Creative Commons Public Domain Dedication waiver (http://creativecommons.org/publicdomain/zero/1.0/) applies to the data made available in this article, unless otherwise stated in a credit line to the data. 
macrophage Th2-type immune response in vitro. Our study suggests that exosome-like vesicles play an important role in the interaction between cysticerci and their hosts.

Keywords: Cysticercus pisiformis, Exosome-like vesicles, Macrophages, Th2-type immune response

\section{Background}

Taenia pisiformis, a common intestinal tapeworm in canines and felines, is widely distributed around the world $[1,2]$. Cysticercus pisiformis, the larval stage of $T$. pisiformis, causes considerable economic losses to the rabbit breeding industry [3]. Infection in the definitive host may occur when they consume lagomorph internal organs infected with T. pisiformis cysticercus. Oryctolagus cuniculus become infected through ingestion of water or forage contaminated with T. pisiformis eggs. Cysticerci usually parasitize the liver capsule, peritoneum, greater omentum and mesentery, and occasionally other organs such as the pelvis or lungs [3, 4]. Rabbits infected with $T$. pisiformis have a weakened immunologic resistance and are susceptible to secondary infections with other pathogens, which may cause host digestive disorders, growth retardation, a decrease in feed conversion, reduction of proliferation, and even death [5].

Helminth infections are generally characterized by a polarized type-2 immune response, which could be initiated by helminth excretory/secretory products (ESP). Recently, helminth-derived exosomes or extracellular vesicles (EVs) have been proven to be a new paradigm in the study of parasite-host communication $[6,7]$. Exosomes are nano-scale $(30-150 \mathrm{~nm})$ membrane-bound vesicles that are released into the extracellular environment via the fusion of the plasma membrane with the multi-vesicular bodies (MVBs) [8-10]. These vesicles are produced by normal [11] and pathological cells [12] and can be obtained from extracellular fluids, including urine [13], blood plasma [14], breast milk [15], saliva [16], and cerebrospinal fluid [17]. Exosomes carry a diverse suite of bioactive molecules, including nucleic acids, proteins and lipids, which can be transferred into target cells to mediate intercellular communication [18-21]. Exosome-like vesicles from helminths may play a pivotal role in parasitic infection [22-25]. Recent studies of several cestodes have revealed that exosome-like vesicles from parasites can deliver miRNAs or proteins cargo into host cells to modulate the host immune system [10, 26]. Echinococcus granulosus cyst fluids contain some parasite-derived EVs and specific proteins, some of which were associated with cyst survival [27]. Echinococcus multilocularis was shown to release exosome-derived miR-71 into the host and affected the function of macrophages [28]. Moreover, Heligmosomoides polygyrus-derived EVs have been shown to be taken up by murine macrophages and inhibited their activation [29]. These studies suggest that exosome-like vesicles take part in parasite-host interactions through delivering specific functional components. However, the information about the contents of cysticercus pisiformis-derived exosome-like vesicles and their functional effects on macrophages remains largely unknown. Therefore, the purpose of the present investigation was to profile the proteins and miRNA components of cysticercus pisiformis-derived exosome-like vesicles and to explore the role of exosomes-like vesicles in macrophages in metacestode infection.

\section{Methods}

\section{Animals and parasites}

Animals used in this study were purchased from the Laboratory Animal Center of Lanzhou Veterinary Research Institute. New Zealand white rabbits were housed individually in wire cages equipped with a plastic nest and had access to food and water ad libitum. Fresh T. pisiformis cysticerci were harvested from New Zealand white rabbits 50 days after infection with 500 eggs of $T$. pisiformis.

\section{Exosome-like vesicles isolation}

To prepare exosome-like vesicles derived from $T$. pisiformis cysticercus, rabbits infected with $T$. pisiformis cysticerci were sedated with xylazine $(5 \mathrm{mg} / \mathrm{kg})$ and ketamine $(25 \mathrm{mg} / \mathrm{kg})$, and euthanized with a lethal dose of sodium pentobarbital $(100 \mathrm{mg} / \mathrm{kg})$. Metacestodes collected from the peritoneum and greater omentum of rabbits were washed thoroughly in sterile $0.9 \%$ sodium chloride containing $100 \mu \mathrm{g} / \mathrm{ml}$ streptomycin and $100 \mathrm{IU} /$ $\mathrm{ml}$ penicillin (Life Technologies, Grand Island, NY, USA). The larvae were washed three times with RPMI-1640 culture medium (Invitrogen, Carlsbad, CA, USA) and maintained in T25 flasks in RPMI-1640 medium supplemented with $10 \%$ exosome-depleted fetal bovine serum (FBS), $100 \mu \mathrm{g} / \mathrm{ml}$ streptomycin and $100 \mathrm{IU} / \mathrm{ml}$ penicillin at $37{ }^{\circ} \mathrm{C}$ under $5 \% \mathrm{CO}_{2}$. Each flask contained 50 cysts in $15 \mathrm{ml}$ culture medium. To ensure host components were expelled thoroughly from larvae, the medium was changed after $12 \mathrm{~h}$ [30]. ESP from T. pisiformis cysticerci were obtained at $24 \mathrm{~h}$ and $48 \mathrm{~h}$ and stored at $4{ }^{\circ} \mathrm{C}$ prior to centrifugation.

Exosome-like vesicles from the ESP of $T$. pisiformis cysticerci were purified by serial centrifugation as previously described [31]. $100 \mathrm{ml}$ pooled ESP from cysticercus 
pisiformis were subjected to two successive centrifugations at $300 \times g$ for $10 \mathrm{~min}$ and $10,000 \times g$ for $30 \mathrm{~min}$ to remove cellular debris and dead cells. The supernatant was harvested and centrifuged at $75,000 \times g$ at $4{ }^{\circ} \mathrm{C}$ for $90 \mathrm{~min}$ to remove large vesicles. The supernatant was collected and centrifuged at $110,000 \times g$ for $90 \mathrm{~min}$ at $4{ }^{\circ} \mathrm{C}$. The resultant pellet was re-suspended in $12 \mathrm{ml}$ filtered PBS, then centrifuged at $110,000 \times g$ for $90 \mathrm{~min}$ to remove remaining protein contaminants, and re-suspended in 50 $\mu \mathrm{l}$ PBS. The concentration of purified exosomal proteins was determined using a Pierce BCA Protein Assay Kit (Thermo Fisher Scientific, Waltham, MA, USA). All aliquots were stored at $-80^{\circ} \mathrm{C}$ until further use.

\section{Transmission electron microscopy (TEM)}

The morphology and size of exosome-like vesicles from cysticercus pisiformis were visualized by TEM. Briefly, 10 $\mu \mathrm{l}$ of exosomes from cysticercus pisiformis were loaded onto a 200-mesh formvar-coated copper grid (Agar Scientific Ltd, Stansted, UK) for $10 \mathrm{~min}$ and the excess stain was removed by blotting with filter paper. Exosome-like vesicle pellets were negatively stained with a $3 \%$ solution of phosphotungstic acid ( $\mathrm{pH}$ 7.0) for $1 \mathrm{~min}$ at room temperature. Grids were air-dried and imaged using a Hitachi TEM at a voltage of $80 \mathrm{kV}$.

\section{Nanoparticle tracking analysis (NTA)}

The size distribution and number of exosome-like vesicles were analyzed by measuring the rate of Brownian motion of each particle using a NanoSight LM10 instrument (Nanosight, Wiltshire, UK). The LM10 uses digital cameras to directly track the movement of individual particles in solution, thereby enabling the determination of particle size distribution as well as the number of nanoparticles [32]. The measurement procedure was performed as previously described [33]. Each sample was measured in triplicate and the NTA analytical software (version 2.3; Nanosight, Wiltshire, UK) was used to capture and analyze the data.

\section{Mass spectrometry analysis}

To identify the proteins of exosome-like vesicles from T. pisiformis cysticercus, three biological replicates were prepared as described above. Each $10 \mu \mathrm{g}$ pelleted exosome was lysed with $150 \mu$ l RIPA lysis buffer (Beyotime, Shanghai, China) and separated by $12 \%$ polyacrylamide gel electrophoresis (PAGE), respectively. All bands were cut into $1 \mathrm{~mm}^{3}$ cubes and washed thrice with $25 \mathrm{mM}$ $\mathrm{NH}_{4} \mathrm{CO}_{3}$ in $50 \%$ acetonitrile (ACN) for $1 \mathrm{~h}$, and subjected to dehydration with $\mathrm{ACN}$ and reduction with 10 $\mathrm{mM}$ dithiothreitol (DTT) at $56^{\circ} \mathrm{C}$ for $1 \mathrm{~h}$. Alkylation was carried by the addition of $55 \mathrm{mM}$ iodoacetamide (IAM) at room temperature for $45 \mathrm{~min}$. In-gel digestion was performed using $2.5 \mu \mathrm{g}$ trypsin at a ratio $(\mathrm{w} / \mathrm{w})$ of $1: 40$ (enzyme:substrate) at $37^{\circ} \mathrm{C}$ overnight and was stopped with $5 \%$ formic acid (FA). Peptides were desalted with a Waters Oasis HLB column (Waters, Milford, MA, USA) and eluted in $2 \% \mathrm{ACN}$ and $0.1 \%$ FA before drying with a Benchtop Centrifugal Vacuum Concentrator (Labconco, Kansas City, MO, USA). Peptides were subjected to LC-20AD nano-HPLC (Shimadzu, Kyoto, Japan) spectrometry for peptide separation and data analysis. Briefly, samples were loaded onto a C18 trap column at $15 \mu \mathrm{l} /$ min in solvent $\mathrm{A}(2 \% \mathrm{ACN}, 0.1 \% \mathrm{FA})$ and the peptides were eluted and loaded onto an analytical column using a 44 min gradient, from $2 \%$ to $35 \%$ solvent B $(98 \% \mathrm{ACN}$, $0.1 \% \mathrm{FA}$ ), at a flow rate of $400 \mathrm{nl} / \mathrm{min}$. The eluate was subjected to nanoelectrospray ionization followed by tandem mass spectrometry in a Q-Exactive Orbitrap mass spectrometer (Thermo Fisher Scientific). Relative parameters were set as a positive ion mode and data dependent mode with full MS scans from 200 to $1800 \mathrm{~m} / \mathrm{z}$, resolution at 70,000 , normalized collision energy at 27 , charge state at $2+, 3+, 4+$ and $5+$, and resolution at 17,500. After the survey scans, the top 15 most abundant precursor ions were fragmented by high-energy collision dissociation (HCD). Since genome information on $T$. pisiformis was not yet obtained, the MS data analysis was carried out by Mascot software (version 2.3.02; Matrix Science, London, UK) using genomes from three parasites that have high kinship with T. pisiformis, including T. solium (http://www.genedb.org/Homepage/T.solium), E. granulosus (ftp://ftp.ncbi.nlm.nih.gov/genomes/all/ GCA/000/524/195/GCA_000524195.1_ASM52419v1/) and E. multilocularis (ftp://ftp.ncbi.nlm.nih.gov/genom es/all/GCA/000/469/725/GCA_000469725.3_EMULT I002/). Additionally, common Repository of Adventitious Proteins (cRAP) (http://www.thegpm.org/crap/) was used to analyze proteins commonly found in proteomic experiments and Oryctolagus cuniculus genome database (https://www.uniprot.org/uniprot/?query=taxon omy:9986) was used to remove the protein from hosts. Database search parameters were set as follows: trypsin as enzyme; peptide mass tolerance of $20 \mathrm{ppm}$ and fragment mass tolerance of $0.05 \mathrm{Da} ;+1,+2,+3$ as peptide charge; a maximum of one missed cleavage; carbamidomethyl (C), iTRAQ8plex (N-term), iTRAQ8plex (K) as fixed modifications and oxidation (M), Gln- > pyro-Glu (N-term $\mathrm{Q})$, deamidated $(\mathrm{NQ})$ as variable modifications. False discovery rate (FDR) lower than 0.01 was used as a screening condition $[34,35]$.

Gene ontology (GO) analysis of the identified proteins was conducted using the Gene ontology database (http:// www.geneontology.org). Functional annotations of the proteins were performed using the Blast2GO program (https://www.blast2go.com) against the non-redundant 
protein database (NCBInr). Additionally, the Clusters of Orthologous Groups (COG) database (http://www.ncbi. nlm.nih.gov/COG/) and Kyoto Encyclopedia of Genes and Genomes (KEGG) database (http://www.genome.jp/ $\mathrm{kegg} /$ ) were used to classify and group these identified proteins.

\section{Western blot analysis}

The protein concentration of exosome-like vesicles, soluble worm antigens (SAg) and ESP from T. pisiformis cysticercus were measured using a BCA protein assay kit. Fifteen $\mu \mathrm{g}$ of total protein was denatured at $100{ }^{\circ} \mathrm{C}$ for 10 min and separated by $12 \%$ SDS-PAGE. The proteins were transferred to polyvinylidene fluoride membranes (Millipore, Burlington, MA, USA) for 13 min and blocked with $5 \%$ non-fat milk in PBST for $2 \mathrm{~h}$ at room temperature. Two antibodies of anti-14-3-3 and anti-enolase (1:200; both from T. solium produced in rabbits and were prepared in our laboratory) [36] were separately added to the membrane and incubated at $4{ }^{\circ} \mathrm{C}$ overnight. The membranes were washed three times with PBST and incubated with HRP-goat-anti rabbit IgG $(\mathrm{H}+\mathrm{L})$ (1:1000; Beyotime, Shanghai, China). The bands were developed using an ECL chemiluminescence working solution (Beyotime) following the manufacturer's instructions.

\section{RNA extraction and high-throughput small RNA sequencing}

Exosome-like vesicles derived from fresh T. pisiformis cysticerci (served as a positive control) in three biological replicates were prepared as described above, and total RNA was extracted using TRIzol reagent (Invitrogen). RNA sample integrity and quality were determined using an Agilent 2100 Bioanalyzer (Agilent Technologies, Santa Clara, CA, USA). High-throughput small RNA sequencing was carried out by BGI (Shenzhen, China). Briefly, RNA fragments (18-30 nt) were separated by PAGE. After ligation of 3' and 5' adaptors to both ends of small RNAs, the ligation products were used for reverse transcription PCR. The PCR products (100-120 bp) were further separated on a PAGE gel and small RNA sequencing libraries were generated using a TruSeq Small RNA Library Preparation Kit (Illumina, San Diego, CA, USA) following the manufacturer's protocol. Library sequencing was carried out on an Illumina HiSeq 2500 system (Illumina), and small RNA clean reads were obtained after removing adaptor reads, low quality reads and contaminants. Because of the unavailability of T. pisiformis genome, we used the T. solium genome (https://parasite.wormbase.org/Taenia_solium_prjna 170813/Info/Index/), E. granulosus (ftp://ftp.ncbi.nlm. nih.gov/genomes/all/GCA/000/524/195/GCA_00052 4195.1_ASM52419v1/) and E. multilocularis (ftp://
ftp.ncbi.nlm.nih.gov/genomes/all/GCA/000/469/725/ GCA_000469725.3_EMULTI002/) as references to align screened small RNA sequences (18-30 nt). Afterwards, the mapped reads were aligned to the miRBase database (http://mirbase.org) and Echinococcus spp. metacestode miRNA dataset to annotate known miRNAs (E-value < 0.05). Small RNA expression profiles, including miRNA, snRNA, snoRNA, tRNA, rRNA, and piRNA, were annotated using the RFam database (http://rfam.janelia.org). The RepBase database (http://www.girinst.org/repba se) and the pre-setting reference genome database were also used to identify small RNAs. In addition, the unannotated sequences were used to predict potential novel miRNA candidates through searching the characteristic hairpin structure of the miRNA precursor [37]. The prediction of targets of exosomal miRNAs was conducted using RNAhybrid, miRanda and TargetScan software. The potential biological functions of target genes were predicted using the KEGG database.

\section{Macrophage cell culture and treatment}

RAW264.7 murine macrophage cell lines were maintained in Dulbecco's modified Eagle's medium (DMEM) supplemented with $10 \%$ FBS and cultured in a $37{ }^{\circ} \mathrm{C}$ incubator with $5 \% \mathrm{CO}_{2}$. The cells were plated in 6-well plates $\left(1 \times 10^{6}\right.$ cells $\left./ \mathrm{ml}\right)$ and incubated with exosomedepleted DMEM conditioned media containing either $200 \mathrm{ng} / \mu \mathrm{l}$ LPS (Sigma-Aldrich, St. Louis, MO, USA), 40 ng/ml IL-4 (Sigma-Aldrich), $50 \mu \mathrm{g} / \mathrm{ml}$ T. pisiformis cysticercus-derived exosome-like vesicles, sterile PBS, or a combination with LPS + exosome-like vesicles, or IL-4 + exosome-like vesicles. All treatments were conducted in triplicate.

\section{Quantitative real-time PCR (qPCR) for miRNAs and mRNAs} miRNAs from $50 \mu \mathrm{l}$ exosome-like vesicles of $T$. pisiformis cysticercus and $20 \mathrm{mg}$ T. pisiformis cysticerci tissue were extracted using an miRNeasy kit (Qiagen, Germantown, $\mathrm{MD}$, USA) following the manufacturer's instructions. The first-strand cDNA was synthesized using $2 \mu \mathrm{g}$ of total miRNA using Mir- $\mathrm{X}^{\mathrm{TM}}$ miRNA First-Strand Synthesis Kit (Takara, Shiga, Japan) according to the manufacturer's protocols. The qPCR reaction system consisted of $12.5 \mu \mathrm{l}$ of $2 \times$ TB Green Advantage Premix, $0.5 \mu$ of $50 \times$ ROX Dye, $0.5 \mu \mathrm{l}$ of miRNA-specific forward primer, $0.5 \mu \mathrm{l}$ of universal miRNA reverse primer, $2 \mu \mathrm{l}$ of cDNA and $9 \mu \mathrm{l}$ of $\mathrm{ddH}_{2} \mathrm{O}$. qPCR reactions were performed on an ABI7500 instrument (Thermo Fisher Scientific, Waltham, MA, USA) according to the following parameters: initial activation at $95^{\circ} \mathrm{C}$ for $30 \mathrm{~s}$ followed by 40 cycles of $95^{\circ} \mathrm{C}$ for 5 $\mathrm{s}$ and $60^{\circ} \mathrm{C}$ for $34 \mathrm{~s}$. Data was evaluated using online software (http://pcrdataanalysis.sabiosciences.com/mirna ). All miRNA primers were purchased from Guangzhou 
RiboBio Co., Ltd (Guangzhou, China) (Additional file 1: Table S1). As a reference, cel-miR-39-3p was added to each sample to monitor miRNA extraction efficiency and normalize sample-to-sample variation. The relative abundance of miRNAs was calculated and normalized using the $2^{-\Delta \Delta \mathrm{Cq}}$ method.

To determine the relative expression of cytokines in RAW264.7 macrophages during T. pisiformis cysticercus exosome-like vesicles stimulation, total RNA from differently treated cells were isolated and probed by qPCR using mouse-specific primers (IL-4, IL-6, IL-10, IL-12, IL-13, Arg-1, iNOS, and the housekeeping gene GAPDH) (Genecopoeia, Guangzhou, China) (Additional file 1: Table S2). The qPCR was conducted using the TransScript Green One-Step qPCR SuperMix (TransGen Biotech, Beijjing, China) on an ABI7500 instrument. The qPCR reaction system consisted of $10 \mu$ of $2 \times$ TransStart Tip Green qPCR SuperMix, $0.4 \mu \mathrm{l}$ of TransScript OneStep RT Enzyme Mix, $0.4 \mu \mathrm{l}$ of Passive Reference Dye, $0.8 \mu \mathrm{l}$ of forward primer, $0.8 \mu \mathrm{l}$ of reverse primer, $2 \mu \mathrm{l}$ of RNA template and $5.6 \mu \mathrm{l}$ of $\mathrm{dd}_{2} \mathrm{O}$. qPCR reaction procedures and data analysis were performed as previously described.

\section{Enzyme-linked immunosorbent assay (ELISA)}

Following RAW264.7 cell stimulation with LPS, IL-4, exosome-like vesicles from $T$. pisiformis cysticercus, PBS, or a combination for $12 \mathrm{~h}, 24 \mathrm{~h}$ and $36 \mathrm{~h}$, the cellfree supernatants were harvested and frozen at $-80{ }^{\circ} \mathrm{C}$ until the assay was performed. The levels of Th1 and Th2 cytokines in the supernatant was assessed using commercially available mouse cytokine (IL-4, IL-6, IL-10, IL-13, IL-12 and IFN- $\gamma$ ) ELISA kits (RayBiotech, Peachtree Corners, GA, USA) according to the manufacturers' protocols. Each experiment was performed in triplicate.

\section{Statistical analyses}

Statistical analyses were conducted using GraphPad Prism 5.0. Comparisons between groups were assessed using the unpaired Student's t-test. Differences among multiple groups were analyzed by a one-way analysis of variance (ANOVA) using SPSS 24.0 (SPSS Inc., Chicago, IL, USA). Data are presented as the mean \pm standard error of the mean (SE). Statistical significance is indicated as ${ }^{*} P<0.05, * P<0.01$ and ${ }^{* * *} P<0.001$.

\section{Results}

Size and morphological analysis of T. pisiformis cysticercus-derived exosome-like vesicles

To confirm the presence of exosome-like vesicles isolated from the culture medium of $T$. pisiformis cysticercus, the pellets obtained from sequential centrifugation were subjected to TEM and NTA analysis. TEM images showed that these vesicles were spherical, approximately 30-150 $\mathrm{nm}$ in diameter and with lipid bilayer-bound membrane structures (Fig. 1a). The particle size distribution of the vesicles was distributed around 50-150 nm and peaked at a mean diameter of $98.47 \mathrm{~nm}$ (Fig. 1b), which had the prototypical size characteristic of exosome-like vesicles and was consistent with exosomes from other parasites $[10,23,27,38-40]$. This data indicated that we successfully isolated and purified exosome-like vesicles from $T$. pisiformis cysticercus.

\section{Characterization of T. pisiformis cysticercus exosomal protein cargo}

The MS/MS analysis identified 87 parasite-unique proteins in $T$. pisiformis cysticercus-derived exosome-like vesicles (Additional file 2: Table S3). GO analysis showed that these proteins were classified into 40 categories by cellular component, biological process, and molecular function (Fig. 2, Additional file 2: Table S4). In terms of the cellular component, the proteins were mostly related to the membrane $(16.06 \%)$, cell part $(16.06 \%)$ and membrane part (11.92\%). Biological process studies suggested that these exosomal proteins were involved in the cellular process (18.39\%), biological regulation (11.49\%) and regulation of biological processes (10.34\%). In addition, most of molecular functions were classified into three categories: binding (40.43\%), catalytic activity (34.04\%) and transporter activity $(9.57 \%)$ in the identified proteins. The top 50 parasite-origin proteins with unique spectra numbers $\geq 3$ are presented in Table 1 , and some of these were identified as the most common exosomal proteins in Exocarta, mainly including chaperones (heatshock protein and beta-soluble NSF attachment protein), cytoskeletal proteins (actin, Rab and tubulin), metabolic enzymes (enolase, phosphoenolpyruvate carboxykinase and fructose 1, 6 bisphosphate aldolase), molecules associated with signal transduction (annexin, 14-3-3 proteins, programmed cell death 6 interacting protein), elongation factor 1-alpha, and phosphoglycerate kinase. Most of these parasite-origin proteins have been described in exosome-like vesicles from Echinococcus and other flatworm parasites [27, 41, 42].

\section{Validation of C. pisiformis exosome-like vesicles proteomics results}

To verify the proteomic data, two exosome-like vesicle enriched proteins (14-3-3 and enolase) were used for confirmation by western blotting. The results showed that these proteins were detected in the exosome-like vesicles, ESP and SAg of T. pisiformis cysticercus (Fig. 3), consistent with the MS result that 14-3-3 and enolase 

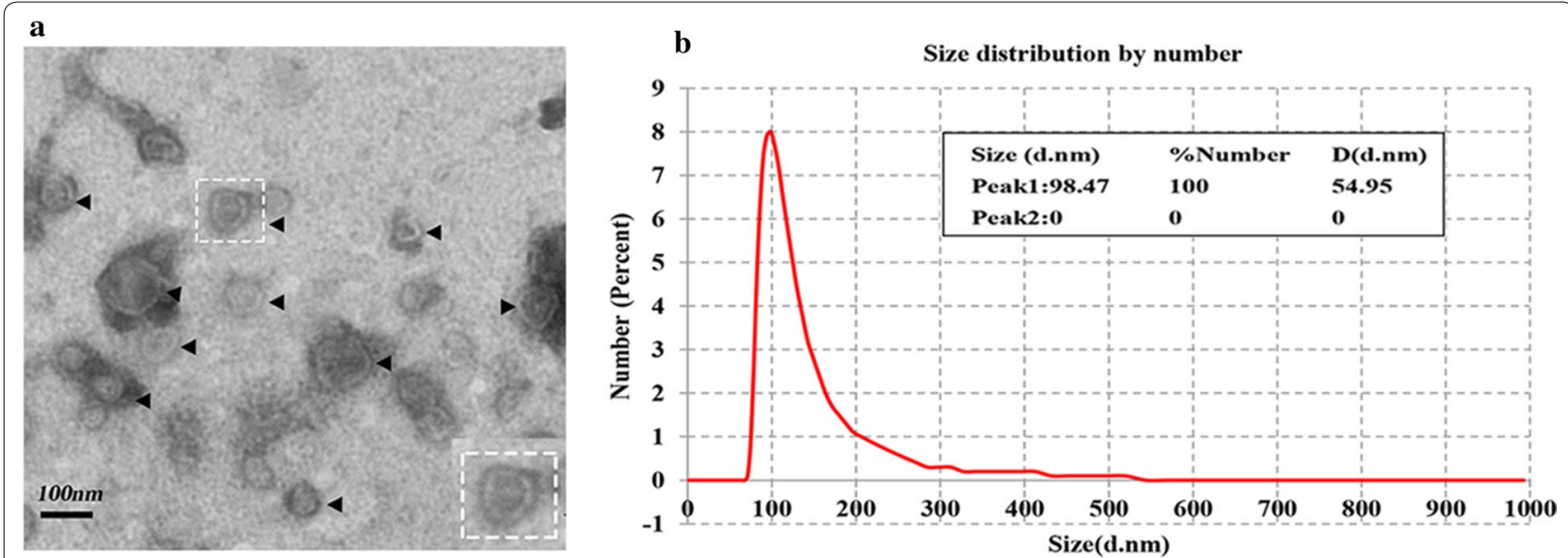

Fig. 1 Characterization of exosome-like vesicles secreted by T. pisiformis cysticercus. a Morphological characterization of T. pisiformis cysticercus exosome-like vesicles by TEM. The arrowheads indicate exosome-like vesicles stained with phosphotungstic acid. The dotted box portion represents a vesicle with a bi-layered membrane. $\mathbf{b}$ NTA diameter distribution analysis of the purified exosome-like vesicles

were enriched in exosome-like vesicles from T. pisiformis cysticercus.

\section{Small RNA sequencing data analysis and $\mathrm{qPCR}$ validation}

To identify the small RNA components in exosomelike vesicles from $T$. pisiformis cysticercus, total RNA extracted from exosome-like vesicles and larvae of T. pisiformis were analyzed using small RNA high-throughput sequencing. The results revealed that a total of $36,961,810$ and 31,592,685 raw reads were identified in exosome-like vesicles and metacestodes from the small RNA sequencing library, respectively. After filtering and processing, a total of 24,329,002 (65.82\%) and 24,897,949 (77.81\%) clean reads were obtained. Among these reads, approximately 14,568,998 (59.88\%) and 6,804,366 (27.66\%) reads were mapped to the reference genome database. All mapped reads were used for small RNA classification, including miRNA, tRNA, rRNA, snRNA and snoRNA (Fig. 4a, b).

A total of 41 and 59 known miRNAs were identified in two libraries. Among them, all of the miRNAs identified in exosome-like vesicles were found in larvae libraries (Fig. 4c, Additional file 3: Table S5, Table S6). The miRNA length distribution of the two libraries showed that almost all of miRNAs were 20-24 nt (Fig. 4d) and the predominant species was $22 \mathrm{nt}$, a typical length of Dicer-processed products, which was consistent with the previous reports in other cestodes [30, 43-45]. Among identified exosomal miRNAs, the most abundant miRNAs were miR-277, followed by miR-10 and miR-71 (Additional file 3: Table S5). Furthermore,
18 novel miRNAs were successfully predicted in the exosome-like vesicles and metacestodes using Mireap (Additional file 3: Table S7).

To validate exosomal small RNA sequencing data, eleven miRNAs with differing abundance were selected for qPCR analysis. Of these miRNAs, the average read counts of miR-10a-5p, miR-219-5p and miR-124b-3p were $28,517,995$ and 130 , respectively, which represented the high, middle and low abundance levels of the identified miRNAs (Additional file 4: Table S5). The quantification results showed that the relative expression levels of 7 known miRNAs (miR-71-5p, miR-10a-5p, miR-let-7-5p, miR-745-3p, miR-219-5p, miR-124-3p and miR-4989-3p) and 4 novel miRNAs (mir-3, mir-7, mir-8 and mir-11) were consistent with those in RNA sequencing (Fig. 5), indicating the accuracy and reliability of the exosomal miRNAs sequencing data.

\section{Bioinformatics analysis of known miRNAs in exosome-like vesicles}

To determine the potential biological functions of the targets of the exosome-like vesicle miRNAs of cysticercus pisiformis, the targets of the exosomal miRNAs were predicted using RNAhybrid and miRanda software packages. A total of 99,278 targets of 41 miRNAs were identified (Additional file 4: Table S8). KEGG analysis revealed that the potential biological functions of most targets were involved mainly in signal transduction and immune system, except for cancer, global and overview maps (Fig. 6). Moreover, some of well-known immune-related miRNAs were identified in exosome-like vesicles, including miR-2a, miR-9, miR-10a, 


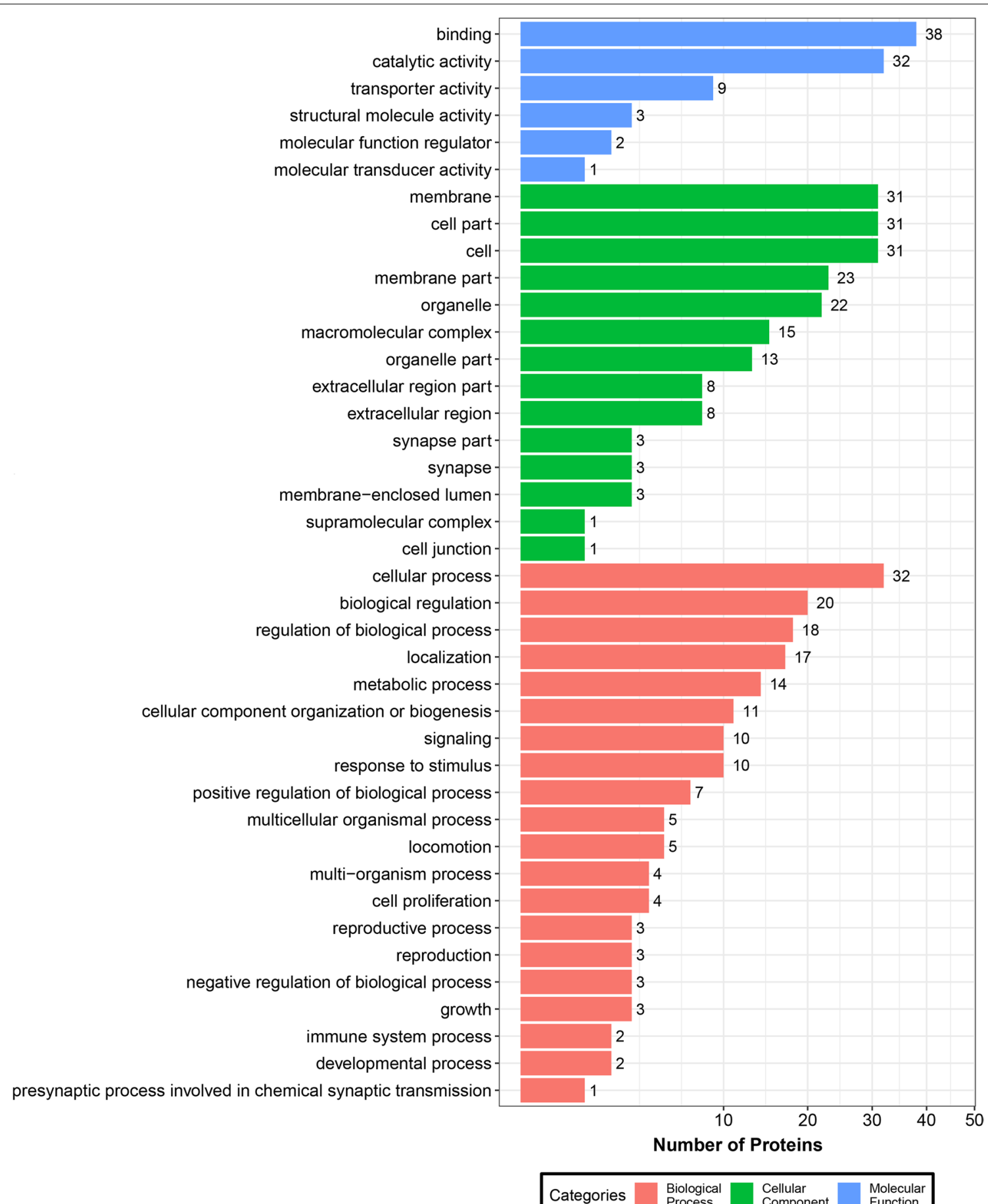

Fig. 2 Functional enrichment analysis of protein cargo enriched in T. pisiformis cysticercus exosome-like vesicles. Bar plot showing biological process, cellular component, and molecular function GO categories in exosome-like vesicles derived from T. pisiformis cysticercus

miR-71 and let-7-5p [23, 28, 46-48]. Therefore, we speculated that the T. pisiformis cysticercus-derived exosomelike vesicles might be involved in modulating host immune response by delivering immune-related miRNA content.
Taenia pisiformis cysticercus exosome-like vesicles stimulated secretion of molecules related to the Th2-type immune response in macrophages

To investigate the potential function of exosome-like vesicles as determined by bioinformatics, we examined 
Table 1 List of top 50 parasite-derived proteins identified in the proteome of exosome-like vesicles from T. pisiformis cysticercus

\begin{tabular}{|c|c|c|c|}
\hline Accession & Annotation & No. of unique peptides & $\begin{array}{l}\text { No. of } \\
\text { unique } \\
\text { spectra }\end{array}$ \\
\hline \multicolumn{4}{|l|}{ Chaperones } \\
\hline EUB56318.1 & Heat shock cognate protein & 6 & 9 \\
\hline EUB64499.1 & Beta-soluble NSF attachment protein & 4 & 4 \\
\hline \multicolumn{4}{|l|}{ Cytoskeletal/Structural proteins } \\
\hline EUB56079.1 & Actin-1 & 9 & 10 \\
\hline EUB64035.1 & Moesin/ezrin/radixin & 7 & 10 \\
\hline EUB54636.1 & Rab & 6 & 9 \\
\hline EUB61143.1 & Tubulin beta-3 chain & 6 & 6 \\
\hline EUB57627.1 & Rab GDP dissociation inhibitor alpha & 4 & 4 \\
\hline \multicolumn{4}{|c|}{ Extracellular matrix/secrected proteins } \\
\hline EUB61528.1 & $\begin{array}{l}\text { Basement membrane-specific heparan sulfate proteoglycan core } \\
\text { protein }\end{array}$ & 5 & 5 \\
\hline EUB57979.1 & Exocyst complex component 3 & 5 & 5 \\
\hline \multicolumn{4}{|l|}{ Hypothetical proteins } \\
\hline EUB54981.1 & Putative phospholipid-transporting ATPase IIB & 13 & 14 \\
\hline EUB54777.1 & Hypothetical protein EGR_10354 & 6 & 6 \\
\hline \multicolumn{4}{|l|}{ Metabolic Enzymes } \\
\hline CDS19796.1 & Enolase & 9 & 9 \\
\hline CDS18138.1 & V type proton ATPase $116 \mathrm{kDa}$ subunit a & 5 & 6 \\
\hline CDS15195.1 & Tryptophanyl tRNA synthetase & 4 & 5 \\
\hline EUB61757.1 & Peptidyl-prolyl cis-trans isomerase & 4 & 5 \\
\hline 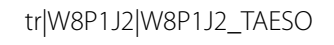 & Phosphoenolpyruvate carboxykinase & 4 & 6 \\
\hline $\operatorname{tr|D2U5C3|D2U5C3\_ TAESO~}$ & Long chain fatty acid coenzyme A ligase 5 & 3 & 4 \\
\hline EUB60416.1 & Long-chain-fatty-acid-CoA ligase & 3 & 4 \\
\hline CDS17202.1 & Fructose 16 bisphosphate aldolase & 3 & 3 \\
\hline \multicolumn{4}{|l|}{ Protease } \\
\hline EUB64462.1 & Calpain-A & 10 & 10 \\
\hline CDS21212.1 & cGMP dependent protein kinase & 9 & 10 \\
\hline CDS24569.1 & Intestinal type alkaline phosphatase 1 & 4 & 4 \\
\hline EUB59398.1 & Phosphoglycerate kinase & 3 & 4 \\
\hline EUB58966.1 & Ras gtpase & 3 & 4 \\
\hline \multicolumn{4}{|c|}{ Signal Transduction and Biological Regulation } \\
\hline EUB64724.1 & Annexin & 6 & 6 \\
\hline EUB62107.1 & 14-3-3 protein & 5 & 5 \\
\hline EUB64038.1 & Vacuolar protein sorting-associated protein $4 \mathrm{~A}$ & 6 & 6 \\
\hline EUB59108.1 & Transforming protein RhoA & 4 & 6 \\
\hline EUB63467.1 & Receptor Mediated Endocytosis family member & 5 & 5 \\
\hline CDS21096.1 & Endophilin B1 & 4 & 5 \\
\hline EUB57999.1 & Ras-related C3 botulinum toxin substrate 2 & 4 & 5 \\
\hline EUB64797.1 & Ras-related protein O-RAL & 4 & 5 \\
\hline EUB63534.1 & Ras-related protein Rap-1b & 4 & 4 \\
\hline EUB64384.1 & Programmed cell death 6 interacting protein & 4 & 4 \\
\hline EUB59848.1 & ADP-ribosylation factor & 4 & 4 \\
\hline EUB60984.1 & Annexin A6 & 3 & 4 \\
\hline \multicolumn{4}{|l|}{ Transporters/Channels } \\
\hline EUB57119.1 & Sodium/potassium-transporting ATPase subunit alpha & 20 & 20 \\
\hline CDS23982.1 & Major vault protein & 9 & 9 \\
\hline EUB60605.1 & Solute carrier family 5 & 5 & 6 \\
\hline
\end{tabular}


Table 1 (continued)

\begin{tabular}{llll}
\hline Accession & Annotation & No. of unique peptides & $\begin{array}{c}\text { No. of } \\
\text { unique } \\
\text { spectra }\end{array}$ \\
\hline CDS22870.1 & Lipid transport protein N terminal & 5 & 6 \\
EUB61207.1 & BRO1 domain containing protein BROX & 4 & 5 \\
EUB62853.1 & Ras-related protein Rab-2A & 4 & 4 \\
EUB62794.1 & Otoferlin & 4 & 4 \\
Others & & 6 & 13 \\
EUB53928.1 & Ubiquitin & 7 & 8 \\
EUB62510.1 & Elongation factor 1-alpha & 6 & 6 \\
EUB62111.1 & Tetracycline resistance leader peptide TetL & 6 & 6 \\
EUB64561.1 & Multidrug resistance protein & 5 & 6 \\
EUB63692.1 & Myoferlin & 4 & 6 \\
EUB59451.1 & NEDD4 E3 ubiquitin protein ligase WWP1 & 3 & 3 \\
EUB59351.1 & Tegumental proteins & 6 \\
\hline
\end{tabular}

Note: Both unique peptide number and unique spectra number $\geq 3$ are listed. Proteins listed in bold font represent the most common proteins of the "top 30 " exosomes-like vesicles in ExoCarta

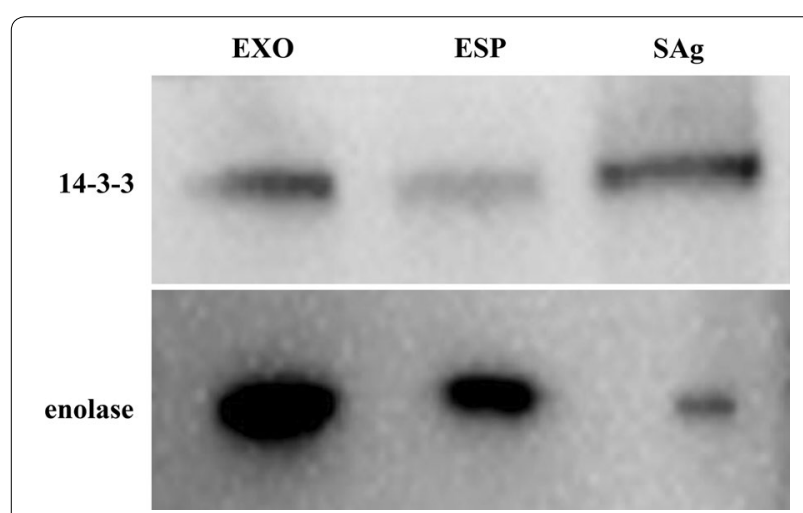

Fig. 3 Western blot of exosomal markers 14-3-3 and enolase. Western blot revealed 14-3-3 with an expected size of approximately $28 \mathrm{kDa}$ and enolase with an expected size of approximately $54 \mathrm{kDa}$. Both ESP and SAg served as positive controls

the in vitro effects of exosome-like vesicles on the release of Th1- and Th2-associated bioactive molecules in RAW264.7 macrophages. The qPCR results showed that the mRNA levels of IFN- $\gamma$ and iNOS at $12 \mathrm{~h}, 24 \mathrm{~h}$ and $36 \mathrm{~h}$ increased significantly in LPS-activated macrophages (Fig. 7a, b, g). The expression of IL-6, IL-10, IL-13 and Arg-1 increased significantly in IL-4-activated macrophages (Fig. $7 \mathrm{~d}-\mathrm{f}, \mathrm{h}$ ). The mRNA levels of IFN- $\gamma$ and iNOS at $12 \mathrm{~h}, 24 \mathrm{~h}$ and $36 \mathrm{~h}$ and IL-12 at 24 $h$ decreased significantly in the LPS + EXO group compared to the LPS group (Fig. 7a, b, g), while expression of Arg- 1 at $12 \mathrm{~h}, 24 \mathrm{~h}$ and $36 \mathrm{~h}, \mathrm{IL}-4$ at $12 \mathrm{~h}$ and $24 \mathrm{~h}, \mathrm{IL}-6$ at $24 \mathrm{~h}, \mathrm{IL}-10$ at $24 \mathrm{~h}$ and IL-13 at $36 \mathrm{~h}$ increased significantly in the LPS + EXO group compared with the LPS group (Fig. 7c-f). These data showed that T. pisiformis cysticercus exosome-like vesicles primed macrophages to secrete Th2 related bioactive molecules.

To further validate the $\mathrm{qPCR}$ results, the supernatant from those groups were collected and used to detect the expression of IL-12, IFN- $\gamma$, IL-4, IL-6, IL-10 and IL-13. The results of ELISA assay showed that both the expression levels of IFN- $\gamma$ and IL-12 at $12 \mathrm{~h}, 24 \mathrm{~h}$ and $36 \mathrm{~h}$ were significantly increased in macrophages after treatment with LPS, while the production of IFN- $\gamma$ and IL-12 at $12 \mathrm{~h}$ and $36 \mathrm{~h}$ decreased significantly in macrophages stimulated with EXO or LPS + EXO compared with LPS treated cells (Fig. 8a, b). Macrophages treated with EXO obviously increased the level of IL-4 secretion at $12 \mathrm{~h}, 24 \mathrm{~h}$ and $36 \mathrm{~h}$. However, cells treated with LPS + EXO produced remarkably lower levels of IL-4 after $24 \mathrm{~h}$ than did cells treated with EXO alone (Fig. 8c). The secretion levels of IL-6, IL-10 and IL-13 at $12 \mathrm{~h}$ and $24 \mathrm{~h}$ were significantly increased in macrophages treated with IL-4 (Fig. 8d-f), and the levels of IL- 6 and IL- 10 at $12 \mathrm{~h}$ and $36 \mathrm{~h}$ were also increased by treatment with EXO compared to the PBS control (Fig. 8d, e).The expression of IL-6 and IL-10 at $24 \mathrm{~h}$ were increased by treatment with IL-4 + EXO compared to the IL-4 control (Fig. 8d, e). The expression of IL-13 at $12 \mathrm{~h}$ had no obvious change after treatment with EXO. However, combination treatment with IL-4 + EXO tended to produce more IL-13 $(P<0.001)$ at $24 \mathrm{~h}$ and $36 \mathrm{~h}$ than did IL-4 treatment alone (Fig. 8f). Taken together, these data suggest that macrophages stimulated by exosome-like vesicles from $T$. pisiformis cysticercus produced mainly Th2 cytokines. 

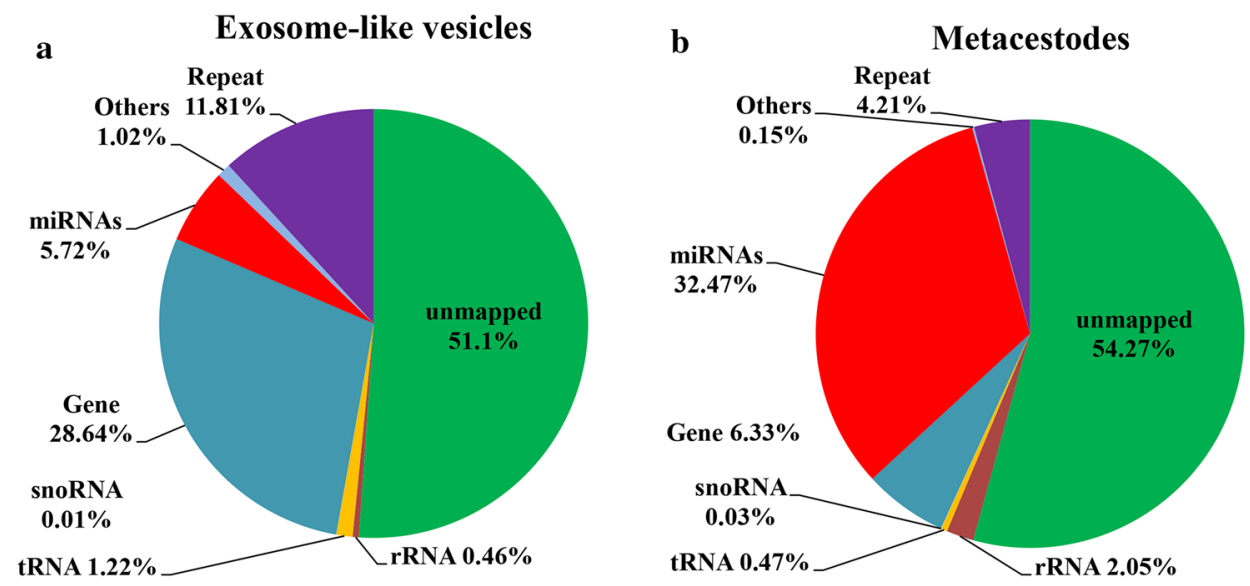

c

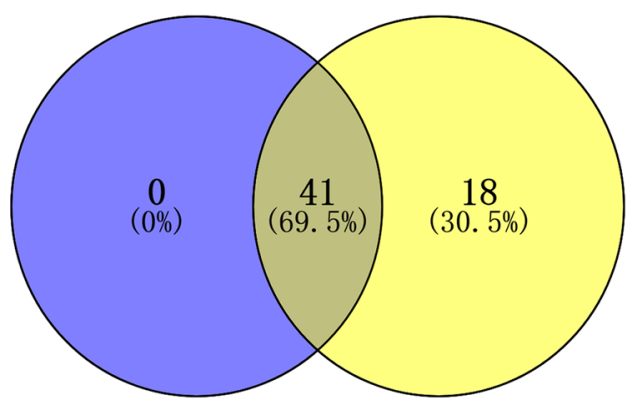

Exosome-like vesicles Metacestodes

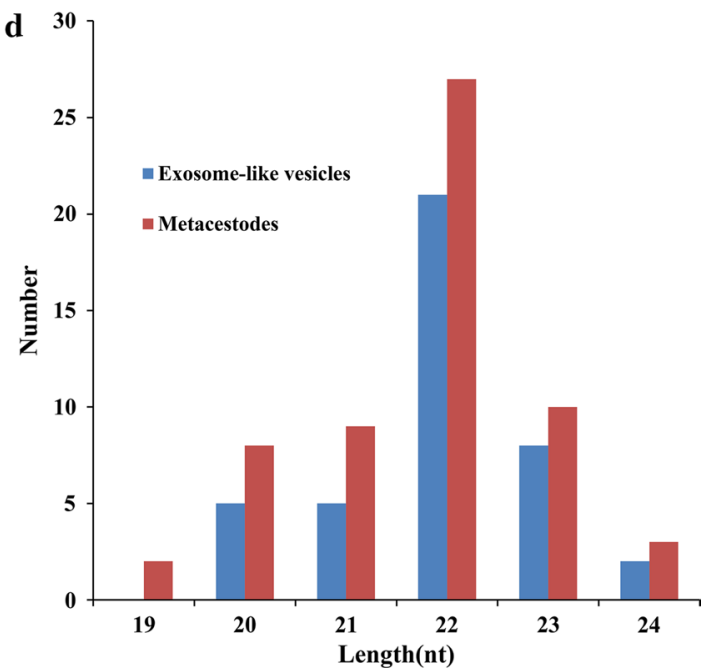

Fig. 4 Length distribution of annotated miRNAs in exosome-like vesicles and cysticercus of T. pisiformis. $\mathbf{a}$, b All the mapped clean reads were annotated, including miRNAs, rRNAs, tRNAs, snRNAs and snoRNAs. Pie-charts of annotated small RNAs and their percentages in exosome-like vesicles and cysticercus of T. pisiformis. c Common miRNAs in exosome-like vesicles and cysticercus of T. pisiformis. $\mathbf{d}$ Length distribution of identified miRNAs

\section{Discussion}

Exosomes, nano-sized endosome-derived membrane vesicles, play vital roles in intercellular communication [49]. An increasing body of studies has revealed exosomes as a ubiquitous molecular mechanism that can transfer bioactive molecules from pathogens to host cells in order to regulate host immune response or promote parasite survival [40]. In the present study, we isolated the exosome-like vesicles derived from T. pisiformis cysticercus, profiled their proteins and miRNAs, and further evaluated their immunomodulatory roles in RAW264.7 macrophages treated with exosome-like vesicles.

We identified seven enzymes involved in energy metabolism (glycolysis, gluconeogenesis and the tricarboxylic acid cycle). Among them, enolase, fructose 1, 6 bisphosphate aldolase and phosphoenolpyruvate carboxykinase are commonly observed in the context of parasite-derived exosome-like vesicles [30, 42]. It is wellknown that, in addition to participating in the glycolysis and gluconeogenesis pathways, enolase can act as a plasminogen receptor as well, which prevents blood clots and facilitates parasite migration within hosts [36], suggesting that enolases packaged within exosome-like vesicles could be important for T. pisiformis cysticercus survival in hosts. Through proteomic analysis, we were able to identify multiple proteins related to exosome biogenesis, including Rabs (Rab-2A, Rab-4A, Rab-6A, Rab-10 and Rab-14), vesicular fusion protein, Vps4, transforming protein RhoA and Rab effectors otoferlin. Most of these molecules have been reported in exosome-like vesicles from Echinococcus and other flatworm parasites [27, 41]. In light of the fact that these proteins are typical molecules associated with the ESCRT-dependent pathway, 

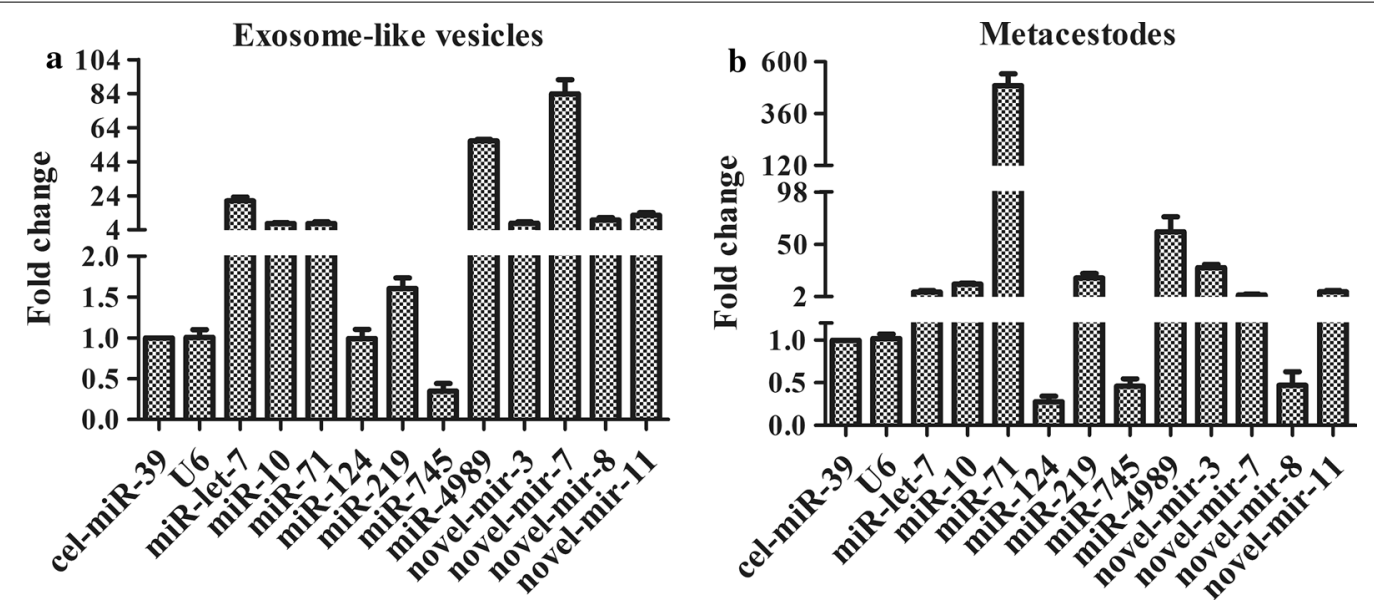

Fig. 5 qPCR identification of relevant expression of miRNAs in exosome-like vesicles and cysticercus of T. pisiformis. a Relevant expression of miRNAs in T. pisiformis cysticercus exosome-like vesicles. b Relevant expression of miRNAs in T. pisiformis cysticercus. Cel-miR-39-3p served as an external control to normalize sample-to-sample variation

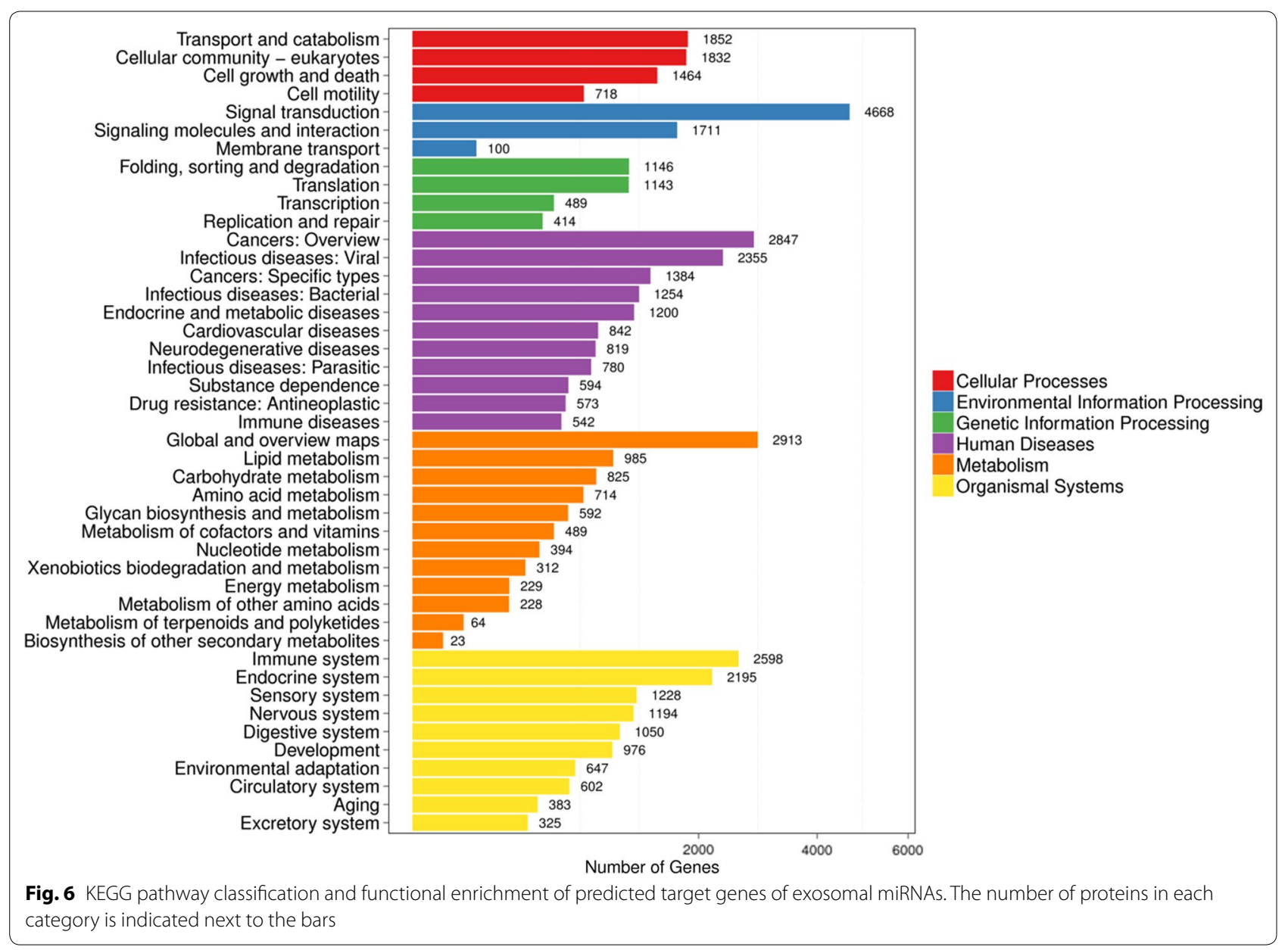



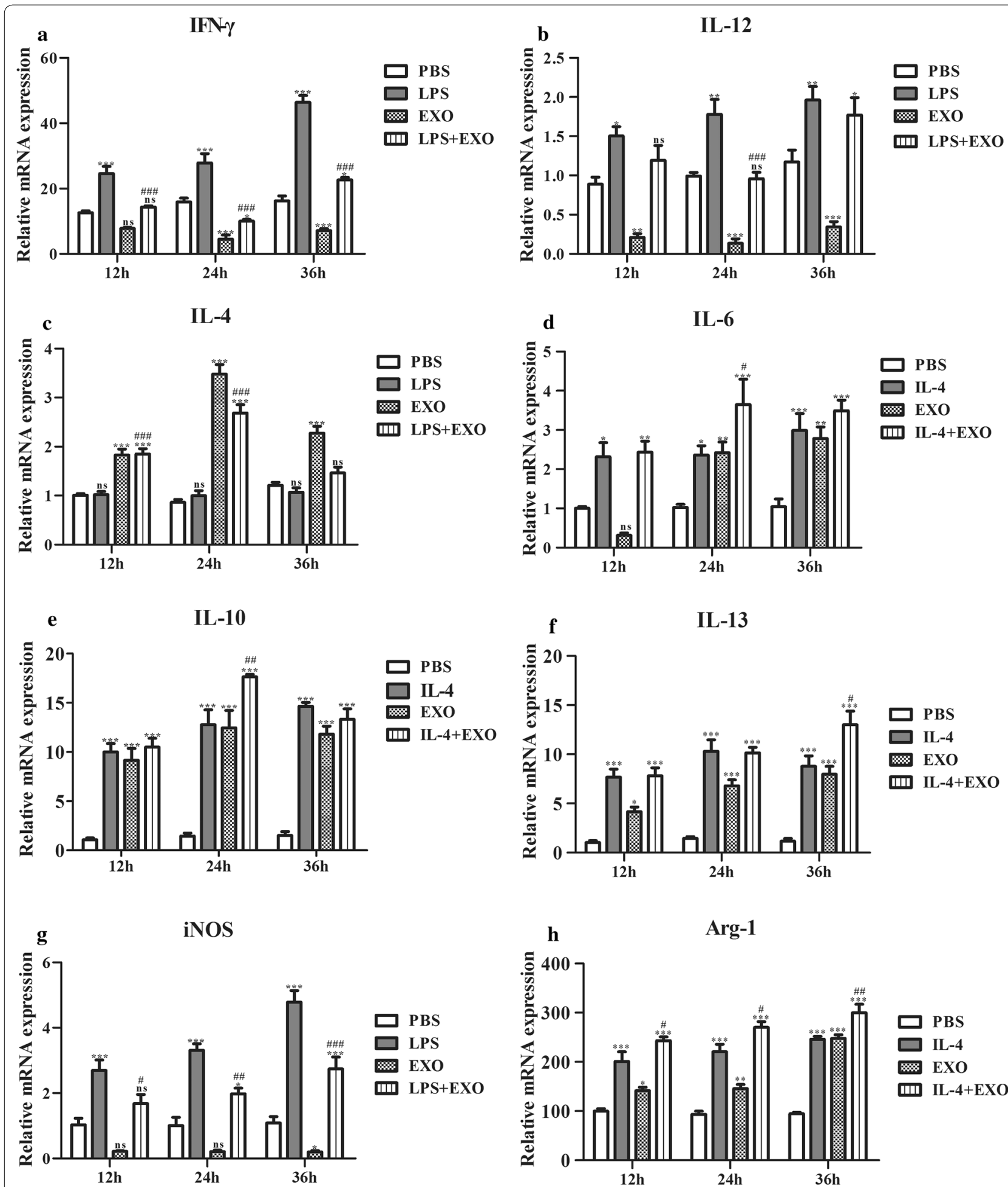

Fig. 7 Fold regulation (qPCR) in M1 and M2 cytokine gene expression induced by T. pisiformis cysticercus exosome-like vesicles in RAW264.7 macrophages. LPS and IL-4 served as positive controls for M1 phenotype and M2 phenotype molecules, respectively. PBS served as the negative control for cytokine stimulaton. $\mathbf{a}, \mathbf{b}, \mathbf{g}$ Induction of M1 markers in RAW264.7 macrophages treated with T. pisiformis cysticercus exosome-like vesicles. $\mathbf{c}-\mathbf{f}, \mathbf{h}$ Induction of M2 markers in RAW264.7 macrophages by T. pisiformis cysticercus exosome-like vesicles. Data for the final analysis are from three independent experiments and are expressed as the mean \pm standard error (SE). ${ }^{*} P<0.05,{ }^{* *} P<0.01$ and ${ }^{* * *} P<0.001$ were considered statistically significant compared to PBS-treated RAW264.7 macrophages. ${ }^{\#} P<0.05,{ }^{\# \#} P<0.01$ and ${ }^{\# \# \#} P<0.001$ were considered statistically significant compared to LPS/L-4 treated RAW264.7 macrophages (Additional file 5: Table S9) 


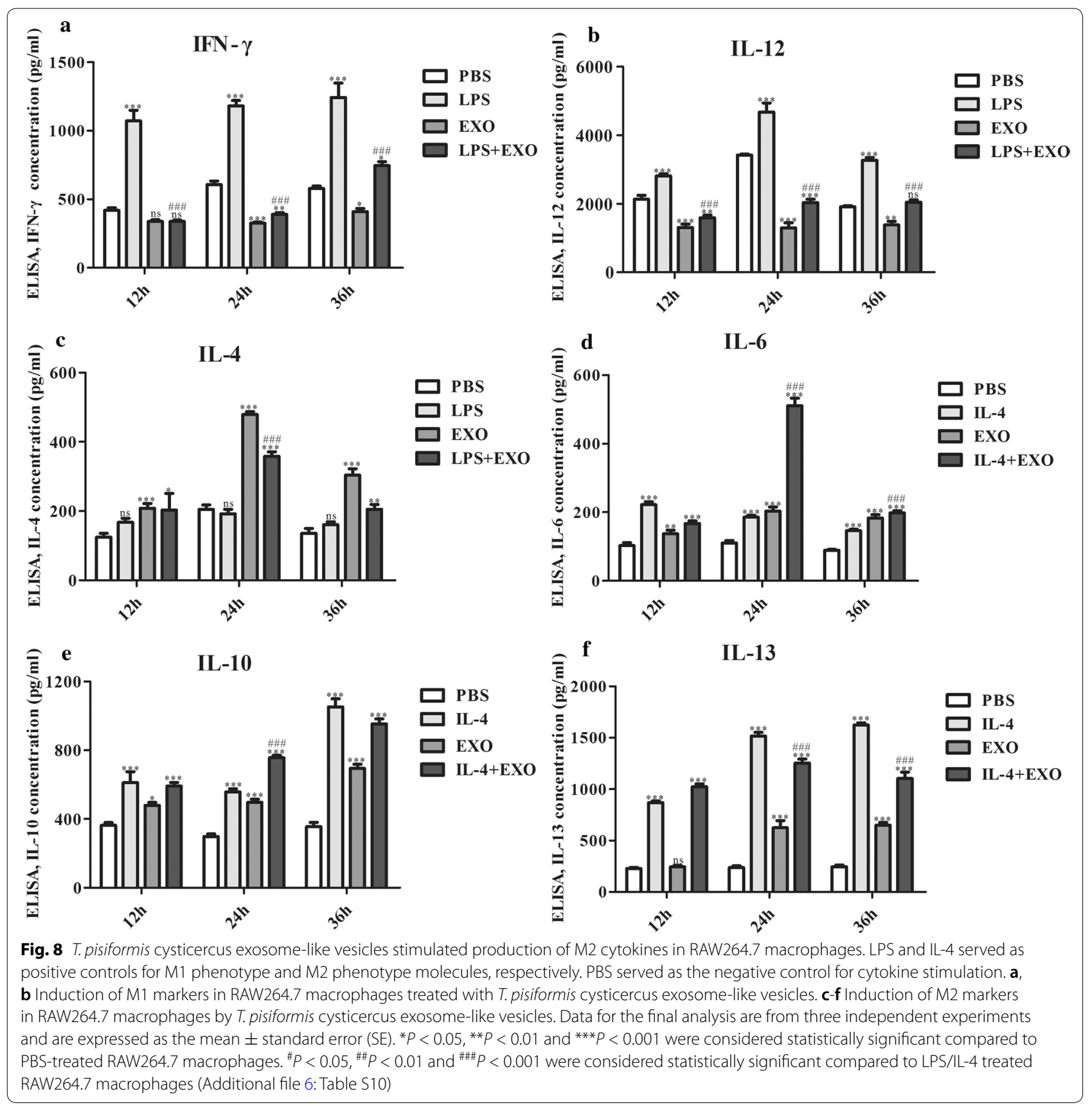

we hypothesized that ESCRT is likely the major route involved in the formation of exosome-like vesicles and the sorting of cargo into exosome-like vesicles, consistent with a previous study of adult Fasciola hepatica [41]. Moreover, the proteomics analysis identified tegumentspecific proteins from T. pisiformis cysticercus exosomelike vesicles, providing evidence for the possible roles of exosome-like vesicles in parasite survival through modification of the host immune response.
We identified 41 known miRNAs in T. pisiformis cysticercus exosome-like vesicles. The biological functions of the predicted targets of these miRNAs were associated mainly with immune system and signal transduction. Some of miRNAs could regulate innate immune response in inflammatory pathways through targeting signaling components. Previous studies suggested that let-7c-5p suppressed LPS-induced inflammation via targeting the DMP1-mediated NF-kB pathway [50]. miR-9 was induced by LPS in human monocytes and 
neutrophils, and increased miR-9 acts as a feedback control of the NF- $\mathrm{B}$ dependent inflammatory response by inhibiting the expression of NFKB1 [51]. Moreover, miR124 and miR-125 have been found to mediate inflammatory response and macrophage activation [52-54]. Thus, transferring these specific miRNAs to host cells might alter certain gene expression in macrophages. Furthermore, qPCR confirmed that eleven selected miRNAs, including seven known miRNAs (miR-71-5p, miR-10a-5p, miR-let-7-5p, miR-745-3p, miR-219-5p, miR-124-3p and miR-4989-3p) and four novel miRNAs (novel-mir-3, novel-mir-7, novel-mir-8 and novel-mir-11) existed in cysticerci and exosome-like vesicles of $T$. pisiformis. Compared to T. pisiformis cysticercus, exosomal novel-mir-7 had the highest relative abundance, followed by miR-let-7-5p, novel-mir-8, novel-mir-11 and miR-124, suggesting that these miRNAs from $T$. pisiformis cysticercus may be selectively encapsulated in exosome-like vesicles. However, little is known about their selective sorting mechanism and it remains to be elucidated in future studies.

The most significant finding of this study was that exosome-like vesicles from $T$. pisiformis cysticercus induced the macrophages polarization toward the M2 phenotype and produced a Th2-type immune response. When RAW264.7 macrophages were treated with $T$. pisiformis cysticercus exosome-like vesicles, the production of Arg1, IL-4, IL-6, IL-10 and IL-13 was significantly increased. In contrast, the expression of iNOS, IFN- $\gamma$ and IL-12 was significantly decreased, revealing that $T$. pisiformis cysticercus exosome-like vesicles participate in promoting macrophages to M2 polarization. There is evidence that injection of exosomes from the intestinal fluke Echinostoma caproni in BALB/c mice prime balanced Th2/Treg immune responses, which alleviates intestinal symptom severity in subsequent challenge infections and benefits parasite survival [55]. The immunomodulatory capacity of exosomes from the murine gastrointestinal nematode, Heligmosomoides polygyrus, has been demonstrated by suppressing innate type 2 lymphoid cell responses. Furthermore, $H$. polygyrus EVs have been shown to suppress the expression of IL1RL1/ST2, the IL-33 receptor, and type 2 innate lymphoid cell responses $[56,57]$. Similarly, our previous studies showed that rabbits immunized with exosomes from $T$. pisiformis cysticercus displayed a higher production of IL-10, which results in decreasing significantly in worm reduction after challenging tapeworm eggs (our unpublished data). These studies suggest that exosome-like vesicles could play an important role in the host Th2-type immune response induced by $T$. pisiformis cysticercus.

In summary, although the presence of exosome-like vesicles has been demonstrated in several parasites, this is the first systemic study on exosome-like vesicles derived from ESP of $T$. pisiformis cysticercus in terms of morphology, size, content and immune regulation. The present work revealed that exosome-like vesicles participated in the process of parasite-host communication and the modulation of host Th2-type immune response induced by $T$. pisiformis cysticercus. The present investigation provides new insights into a deep understanding of molecular cargo in exosome-like vesicles of $T$. pisiformis cysticercus and the pathogenesis of exosome-like vesicle-mediated metacestodiasis.

\section{Conclusions}

We successfully purified exosome-like vesicles from $T$. pisiformis cysticercus and profiled their protein and miRNA components, demonstrating the potential biological functions of exosome-like vesicles in the host immune response. Interestingly, the present study reveals the upregulation of molecules associated with the Th2type immune response in RAW264.7 macrophages after stimulation with exosome-like vesicles from $T$. pisiformis cysticercus, which might facilitate survival of $T$. pisiformis cysticercus in rabbits. Further exploration of exosomal miRNA targets will be beneficial to elucidate the immunomodulatory mechanism and the important role of exosome-like vesicles in the interaction between the host and T. pisiformis cysticercus.

\section{Supplementary information}

Supplementary information accompanies this paper at https://doi. org/10.1186/s13071-020-04186-z.

Additional file 1: Table S1. Primers for miRNAs qPCR validation. Table S2. Primers for $\mathrm{qPCR}$ of Th1/Th2 associated molecules.

Additional file 2: Table S3. Protein identification of exosome-like vesicles from T. pisiformis cysticercus. Table S4. GO analysis of exosomal proteins from T. pisiformis cysticercus.

Additional file 3: Table S5. miRNAs identified in exosome-like vesicles from T. pisiformis cysticercus. Table S6. miRNAs identified in T. pisiformis cysticercus. Table S7. Newly discovered miRNAs in exosome-like vesicles and T. pisiformis cysticercus.

Additional file 4: Table S8. The potential targets of T. pisiformis cysticercus exosomal miRNAs.

Additional file 5: Table S9. Results of statistical analyses of qPCR.

Additional file 6: Table S10. Results of statistical analyses of ELISA.

\footnotetext{
Abbreviations

ACN: acetonitrile; Arg-1: arginase-1; DTT: dithiothreitol; ESP: excretory/secretory products; EVs: extracellular vesicles; FBS: fetal bovine serum; GO: Gene Ontology; HCD: high-energy collision dissociation; IAM: iodoacetamide; IFN-ү: interferon-ү; IL-4: interleukin-4; IL-6: interleukin-6; IL-10: interleukin-10; IL-13: interleukin-13; KEGG: Kyoto Encyclopedia of Genes and Genomes; LPS: lipopolysaccharide; M1: classically activated macrophages; M2: alternatively activated macrophages; MVBs: multi-vesicular bodies; NTA: nanoparticle tracking analysis; PAGE: polyacrylamide gel electrophoresis;
} 
PBS: phosphate-bufered saline; RPMI 1640: Roswell Park Memorial Institute 1640 culture media; SAg: soluble worm antigens; TEM: transmission electron microscope

\section{Acknowledgements}

The authors are thankful for the technical assistance provided by BGI-Tech, BGI, Shenzhen, PR China.

\section{Authors' contributions}

XNL and LQW designed the experiments and drafted the manuscript. LQW, $\mathrm{LM}$ and $\mathrm{SHZ}$ performed the experiments and conducted the data analysis. LQW, SHZ, TSL, PHL, YPL and GXL conducted the bioinformatics analyses. LQW, $T L L$ and $P H L$ drafted the initial version of the manuscript. $X N L, T L L$ and $M L$ drafted the final version of the manuscript. All authors read and approved the final manuscript.

\section{Funding}

This research was supported by grants from the National Key Research and Development Program of China (No. 2017 YFD0501303 and No. 2017YFC1601206), the National Natural Science Foundation of China (No. 31772726 and No.31372433) and the Central Public-interest Scientific Institution Basal Research Fund (No. 1610312020017).

\section{Availability of data and materials}

Data supporting the conclusions of this article are included within the article and its additional file.

\section{Ethics approval and consent to participate}

All animal protocols were approved by the Animal Administration and Ethics Committee of Lanzhou Veterinary Research Institute, Chinese Academy of Agricultural Sciences (Permit No. LVRIAEC-2009-006). The study was performed in accordance with good animal practices set forth in the Animal Ethics Procedures and Guidelines of the People's Republic of China.

\section{Consent for publication}

Not applicable.

\section{Competing interests}

The authors declare that they have no competing interests.

\section{Author details}

1 State Key Laboratory of Veterinary Etiological Biology, Key Laboratory of Veterinary Parasitology of Gansu Province, Lanzhou Veterinary Research Institute, Chinese Academy of Agricultural Sciences, Lanzhou 730046, Gansu Province, People's Republic of China. ${ }^{2}$ Jiangsu Co-Innovation Center for the Prevention and Control of Important Animal Infectious Disease and Zoonoses, Yangzhou University, Yangzhou 225009, People's Republic of China.

Received: 22 February 2020 Accepted: 13 June 2020

Published online: 19 June 2020

\section{References}

1. Richards DT, Haris S, Lewis JW. Epidemiological studies on intestinal helminth parasites of rural and urban red foxes (Vulpes vulpes) in the United Kingdom. Vet Parasitol. 1995;59:39-51.

2. Smith GC, Gangadharan B, Taylor Z, Laurenson MK, Bradshaw H, Hide G, et al. Prevalence of zoonotic important parasites in the red fox (Vulpes vulpes) in Great Britain. Vet Parasitol. 2003;118:133-42.

3. Hallal-Calleros C, Morales-Montor J, Orihuela-Trujillo A, Togno-Peirce C, Murcia-Mejia C, Bielli A, et al. Taenia pisiformis cysticercosis induces decreased prolificacy and increased progesterone levels in rabbits. Vet Parasitol. 2016;229:50-3.

4. Dominguez-Roldan R, Perez-Martinez M, Rosetti MF, Arias-Hernandez D, Bernal-Fernandez G, Flores-Perez Fl, et al. High frequency of Taenia pisiformis metacestodes and high sex-associated susceptibility to cysticercosis in naturally infected wild rabbits. Parasitol Res. 2018;117:2201-6.
5. Rajasekariah GR, Rickard MD, O'Donnell IJ. Taenia pisiformis: protective immunization of rabbits with solubilized oncospheral antigens. Exp Parasitol. 1985;59:321-7.

6. Coakley G, Maizels RM, Buck AH. Exosomes and other extracellular vesicles. The new communicators in parasite infections. Trends Parasitol. 2015;31:477-89.

7. Wu Z, Wang L, Li J, Wang L, Wu Z, Sun X. Extracellular vesicle-mediated communication within host-parasite interactions. Front Immunol. 2018:9:3066.

8. Jorgensen M, Baek R, Pedersen S, Sondergaard EK, Kristensen SR, Varming K. Extracellular vesicle (EV) array: microarray capturing of exosomes and other extracellular vesicles for multiplexed phenotyping. J Extracell Vesicles. 2013;2:20920.

9. Tkach $\mathrm{M}$, Thery $\mathrm{C}$. Communication by extracellular vesicles: where we are and where we need to go. Cell. 2016;164:1226-32.

10. Nicolao MC, Rodriguez Rodrigues C, Cumino AC. Extracellular vesicles from Echinococcus granulosus larval stage: isolation, characterization and uptake by dendritic cells. PLoS Negl Trop Dis. 2019;13:e0007032.

11. Mitchell MD, Peiris HN, Kobayashi M, Koh YQ, Duncombe G, Illanes SE, et al. Placental exosomes in normal and complicated pregnancy. Am J Obstet Gynecol. 2015;213:S173-81.

12. Wolfers J, Lozier A, Raposo G, Regnault A, Thery C, Masurier C, et al. Tumor-derived exosomes are a source of shared tumor rejection antigens for CTL cross-priming. Nat Med. 2001;7:297-303.

13. Pisitkun T, Shen RF, Knepper MA. Identification and proteomic profiling of exosomes in human urine. Proc Natl Acad Sci USA. 2004;101:13368-73.

14. Caby MP, Lankar D, Vincendeau-Scherrer C, Raposo G, Bonnerot C. Exosomal-like vesicles are present in human blood plasma. Int Immunol. 2005;17:879-87.

15. Admyre C, Johansson SM, Qazi KR, Filen JJ, Lahesmaa R, Norman M, et al. Exosomes with immune modulatory features are present in human breast milk. J Immunol. 2007;179:1969-78.

16. Lasser C, Alikhani VS, Ekstrom K, Eldh M, Paredes PT, Bossios A, et al. Human saliva, plasma and breast milk exosomes contain RNA: uptake by macrophages. J Transl Med. 2011;9:9.

17. Vella LJ, Greenwood DL, Cappai R, Scheerlinck JP, Hill AF. Enrichment of prion protein in exosomes derived from ovine cerebral spinal fluid. Vet Immunol Immunopathol. 2008;124:385-93.

18. Schatz D, Vardi A. Extracellular vesicles-new players in cell-cell communication in aquatic environments. Curr Opin Microbiol. 2018;43:148-54.

19. Marcilla A, Martin-Jaular L, Trelis M, de Menezes-Neto A, Osuna A, Bernal $D$, et al. Extracellular vesicles in parasitic diseases. J Extracell Vesicles. 2014;3:25040.

20. Thery C. Exosomes: secreted vesicles and intercellular communications. F1000 Biol Rep. 2011;3:15.

21. Lotvall J, Hill AF, Hochberg F, Buzas El, Di Vizio D, Gardiner C, et al. Minimal experimental requirements for definition of extracellular vesicles and their functions: a position statement from the International Society for Extracellular Vesicles. J Extracell Vesicles. 2014;3:26913.

22. Wang L, Li Z, Shen J, Liu Z, Liang J, Wu X, et al. Exosome-like vesicles derived by Schistosoma japonicum adult worms mediates $\mathrm{M} 1$ type immune- activity of macrophage. Parasitol Res. 2015;114:1865-73.

23. Buck AH, Coakley G, Simbari F, McSorley HJ, Quintana JF, Le Bihan T, et al. Exosomes secreted by nematode parasites transfer small RNAs to mammalian cells and modulate innate immunity. Nat Commun. 2014;5:5488.

24. Bernal D, Trelis M, Montaner S, Cantalapiedra F, Galiano A, Hackenberg M, et al. Surface analysis of Dicrocoelium dendriticum. The molecular characterization of exosomes reveals the presence of miRNAs. J Proteomics. 2014;105:232-41.

25. Tritten L, Geary TG. Helminth extracellular vesicles in host-parasite interactions. Curr Opin Microbiol. 2018;46:73-9.

26. Ancarola ME, Marcilla A, Herz M, Macchiaroli N, Perez M, Asurmendi S, et al. Cestode parasites release extracellular vesicles with microRNAs and immunodiagnostic protein cargo. Int J Parasitol. 2017:47:675-86.

27. Siles-Lucas M, Sanchez-Ovejero C, Gonzalez-Sanchez M, Gonzalez E, Falcon-Perez JM, Boufana B, et al. Isolation and characterization of exosomes derived from fertile sheep hydatid cysts. Vet Parasitol. 2017;236:22-33.

28. Zheng Y, Guo X, He W, Shao Z, Zhang X, Yang J, et al. Effects of Echinococcus multilocularis miR-71 mimics on murine macrophage RAW264.7 cells. Int Immunopharmacol. 2016;34:259-62. 
29. Coakley G, McCaskill JL, Borger JG, Simbari F, Robertson E, Millar M, et al. Extracellular vesicles from a helminth parasite suppress macrophage activation and constitute an effective vaccine for protective immunity. Cell Rep. 2017;19:1545-57.

30. Liang P, Mao L, Zhang S, Guo X, Liu G, Wang L, et al. Identification and molecular characterization of exosome-like vesicles derived from the Taenia asiatica adult worm. Acta Trop. 2019;198:105036.

31. Ramakrishnaiah V, Thumann C, Fofana I, Habersetzer F, Pan Q, de Ruiter $\mathrm{PE}$, et al. Exosome-mediated transmission of hepatitis $\mathrm{C}$ virus between human hepatoma Huh7.5 cells. Proc Natl Acad Sci USA. 2013;110:13109-13.

32. Wilson DR, Green JJ. Nanoparticle tracking analysis for determination of hydrodynamic diameter, concentration, and zeta-potential of polyplex nanoparticles. Methods Mol Biol. 2017;1570:31-46.

33. Chan MY, Dowling QM, Sivananthan SJ, Kramer RM. Particle sizing of nanoparticle adjuvant formulations by dynamic light scattering (DLS) and nanoparticle tracking analysis (NTA). Methods Mol Biol. 2017;1494:239-52.

34. Wang W, Zhou X, Cui F, Shi C, Wang Y, Men Y, et al. Proteomic analysis on exosomes derived from patients's Sera infected with Echinococcus granulosus. Korean J Parasitol. 2019;57:489-97.

35. Guan S, Yu H, Yan G, Gao M, Sun W, Zhang X. Characterization of urinary exosomes purified with size cxclusion chromatography and ultracentrifugation. J Proteome Res. 2020;19:2217-25.

36. Zhang S, Guo A, Zhu X, You Y, Hou J, Wang Q, et al. Identification and functional characterization of alpha-enolase from Taenia pisiformis metacestode. Acta Trop. 2015;144:31-40.

37. Friedlander MR, Chen W, Adamidi C, Maaskola J, Einspanier R, Knespel S, et al. Discovering microRNAs from deep sequencing data using miRDeep. Nat Biotechnol. 2008;26:407-15.

38. Santos GB, Monteiro KM, da Silva ED, Battistella ME, Ferreira HB, Zaha A. Excretory/secretory products in the Echinococcus granulosus metacestode: is the intermediate host complacent with infection caused by the larval form of the parasite? Int J Parasitol. 2016;46:843-56.

39. Gillan V, Simpson DM, Kinnaird J, Maitland K, Shiels B, Devaney E. Characterisation of infection associated microRNA and protein cargo in extracellular vesicles of Theileria annulata infected leukocytes. Cell Microbiol. 2019:21:e12969.

40. Samoil V, Dagenais M, Ganapathy V, Aldridge J, Glebov A, Jardim A, et al. Vesicle-based secretion in schistosomes: analysis of protein and microRNA (miRNA) content of exosome-like vesicles derived from Schistosoma mansoni. Sci Rep. 2018;8:3286.

41. Cwiklinski K, de la Torre-Escudero E, Trelis M, Bernal D, Dufresne PJ, Brennan GP, et al. The extracellular vesicles of the helminth pathogen, Fasciola hepatica: biogenesis pathways and cargo molecules involved in parasite pathogenesis. Mol Cell Proteomics. 2015;14:3258-73.

42. Nawaz M, Malik MI, Hameed M, Zhou J. Research progress on the composition and function of parasite-derived exosomes. Acta Trop. 2019;196:30-6.

43. Zheng Y. High-throughput identification of miRNAs of Taenia ovis, a cestode threatening sheep industry. Infect Genet Evol. 2017;51:98-100.

44. Guo X, Zheng Y. Identification and characterization of microRNAs in a cestode Hydatigera taeniaeformis using deep sequencing approach. Exp Parasitol. 2018;194:32-7.

45. Wu J, Yang J, He G, Guo X, Kutyrev I, Kandil OM, et al. High-throughput identification of microRNAs in Taenia hydatigena, a cestode threatening livestock breeding industry. Infect Genet Evol. 2019;75:103985.
46. Pham TT, Ban J, Lee K, Hong Y, Lee J, Truong AD, et al. MicroRNA gga-miR10a-mediated transcriptional regulation of the immune genes in necrotic enteritis afflicted chickens. Dev Comp Immunol. 2020;102:103472.

47. Essandoh K, Li Y, Huo J, Fan GC. MiRNA-mediated macrophage polarization and its potential role in the regulation of inflammatory response. Shock. 2016;46:122-31.

48. Banerjee $\mathrm{S}$, Xie N, Cui H, Tan Z, Yang S, Icyuz M, et al. MicroRNA let-7c regulates macrophage polarization. J Immunol. 2013;190:6542-9.

49. Ranjbaran A, Latifi Z, Nejabati HR, Abroon S, Mihanfar A, Sadigh AR, et al. Exosome-based intercellular communication in female reproductive microenvironments. J Cell Physiol. 2019;234:19212-22.

50. Yuan $\mathrm{H}$, Zhang H, Hong L, Zhao H, Wang J, Li H, et al. MicroRNA let-7c-5p suppressed lipopolysaccharide-induced dental pulp inflammation by inhibiting dentin matrix protein-1-mediated nuclear factor kappa b (NFKB) pathway in vitro and in vivo. Med Sci Monit. 2018;24:6656-65.

51. Bazzoni F, Rossato M, Fabbri M, Gaudiosi D, Mirolo M, Mori L, et al. Induction and regulatory function of miR-9 in human monocytes and neutrophils exposed to proinflammatory signals. Proc Natl Acad Sci USA. 2009;106:5282-7.

52. Chaudhuri AA, So AY, Sinha N, Gibson WS, Taganov KD, O'Connell RM, et al. MicroRNA-125b potentiates macrophage activation. J Immunol. 2011;187:5062-8

53. Ponomarev ED, Veremeyko T, Barteneva N, Krichevsky AM, Weiner HL. MicroRNA-124 promotes microglia quiescence and suppresses EAE by deactivating macrophages via the C/EBP-alpha-PU.1 pathway. Nat Med. 2011;17:64-70

54. Yao L, Ye Y, Mao H, Lu F, He X, Lu G, et al. MicroRNA-124 regulates the expression of MEKK3 in the inflammatory pathogenesis of Parkinson's disease. J Neuroinflammation. 2018;15:13.

55. Trelis M, Galiano A, Bolado A, Toledo R, Marcilla A, Bernal D. Subcutaneous injection of exosomes reduces symptom severity and mortality induced by Echinostoma caproni infection in BALB/C mice. Int J Parasitol. 2016:46:799-808

56. Nowacki FC, Swain MT, Klychnikov OI, Niazi U, Ivens A, Quintana JF, et al. Protein and small non-coding RNA-enriched extracellular vesicles are released by the pathogenic blood fluke Schistosoma mansoni. J Extracell Vesicles. 2015;4:28665.

57. McSorley HJ, Blair NF, Smith KA, McKenzie AN, Maizels RM. Blockade of IL-33 release and suppression of type 2 innate lymphoid cell responses by helminth secreted products in airway allergy. Mucosal Immunol. 2014;7:1068-78.

\section{Publisher's Note}

Springer Nature remains neutral with regard to jurisdictional claims in published maps and institutional affiliations.

Ready to submit your research? Choose BMC and benefit from

- fast, convenient online submission

- thorough peer review by experienced researchers in your field

- rapid publication on acceptance

- support for research data, including large and complex data types

- gold Open Access which fosters wider collaboration and increased citations

- maximum visibility for your research: over $100 \mathrm{M}$ website views per year

At BMC, research is always in progress.

Learn more biomedcentral.com/submissions 